\title{
Solving few-body scattering problems in the momentum lattice basis
}

\author{
O.A. Rubtsova ${ }^{a}$, V.I. Kukulin, and V.N. Pomerantsev \\ Institute of Nuclear Physics, Moscow State University, 119991 Moscow, Russia
}

\begin{abstract}
The brief description of a new approach based on the Wave-Packet Continuum Discretization method recently developed by the present authors towards solving few-body quantum scattering problems is given. The formalism uses the complete continuum discretization scheme in terms of the momentum stationary wave-packet basis, which leads to formulation of the scattering problem on a lattice in the momentum space. The solution of the few-body scattering problem can be found in the approach from linear matrix equations with non-singular matrix elements, averaged on energy over lattice cells.
\end{abstract}

\section{Introduction}

For last decades a lot of different methods for treatment of the few-body scattering problems have been developed. Along with effective direct techniques for solving Faddeev and Faddeev-Yakubovsky scattering equations [1-4], many alternative approaches using $L_{2}$ type wave functions for a description of processes in continuum have been proposed [5-13]. Nowadays such $L_{2}$ methods become very actual because most of them allow to formulate the scattering problems in terms of matrix equations and make the solution of few-body scattering to be quite similar to the treatment of bound state problems. So the development of $L_{2}$-type techniques is a very perspective way on which the few-body scattering problems could be treated most effectively and with the usage of even modern PC instead of powerful supercomputers. However the main part of existing $L_{2}$ methods could not be treated as universal $L_{2}$ formalism for the quantum few-body scattering theory and most of them are used in particular cases only.

Few years ago the present authors have developed a new approach to solving few-body scattering problems based on the complete discretization of few-body continuous spectrum [14-17]. We constructed universal formalism for such a discretization, which uses the stationary wave packets, i.e. $L_{2}$ functions, instead of the exact scattering wave functions. This leads to formulation of the scattering theory on the lattice in the momentum space. In such an approach, finite-dimensional (f.-d.) approximations for basic scattering-theory operators have been obtained and matrix analogs for the scattering equations have been constructed. The approach have recently been tested for the elastic scattering and breakup of a composite projectiles scattered off heavy targets (with neglecting the stripping processes), where a perfect agreement with the conventional Continuum Discretized Coupled Channel method (CDCC) method have been found [14]. Further, the method has been successfully applied to the solution of the Fad-

\footnotetext{
a e-mail: rubtsova-olga@yandex.ru
}

deev equations for $n-d$ scattering above the three-nucleon breakup threshold [16]. Very recently some new method the Discrete Spectral Shift (DSS) formalism has been developed on the base of the lattice approach. This approach allows to find observables for the multi-channel scattering problems from the one-fold diagonalization of the Hamiltonian matrix without solution of any scattering equations [18].

Here we briefly outline main features and results of the wave-packet approach. The present paper is organized as follows. In the section II we introduce the generalized stationary wave-packet basis and describe different ways how to extract scattering information from the total Hamiltonian matrix in such a basis. We define the DSS formalism and construct the total resolvent f.-d. representation. In the section III, we generalize the above technique to the solution of multi-channel scattering problem. Threeand few-body lattice bases are defined in the Section IV, where wave-packet scheme for solving general three- and few-body problems is derived. Brief conclusion is given in Section V.

\section{Two-body scattering problem}

\subsection{Introduction of the lattice basis}

Let's define the lattice wave-packet basis corresponding to the discretization of the continuous spectrum of the free Hamiltonian $h_{0}$. By the discretization procedure, the continuum of $h_{0}$ is confined within the maximal value $E_{\max }$ and the interval $\left[0, E_{\max }\right]$ is divided into a finite number of non-overlapping energy bins $\left[\epsilon_{i-1}, \epsilon_{i}\right]_{i=1}^{N}$ (with $E_{0}=0$ and $\left.E_{N}=E_{\max }\right)^{1}$ with widths $D_{i} \equiv \epsilon_{i}-\epsilon_{i-1}$. Each such energy bin corresponds to the momentum (or wave number) interval $\left[q_{i-1}, q_{i}\right]$ with the width $d_{i}=q_{i}-q_{i-1}$ on momentum axis $q$, where $q=\sqrt{2 \mu \epsilon}$ and $\mu$ is the reduced mass.

\footnotetext{
${ }^{1}$ Here we assume that the value $E_{\max }$ is sufficiently large to provide a proper solution of the discussed problem.
} 
Consider further a complete set of the $h_{0}$ continuum states $\left|\psi_{0 q}\right\rangle$ (plane waves), which are normalized according to the Dirac $\delta$-function on the momentum value $q$. The set of free stationary wave packets (WPs) is defined as integrals of the plane waves (corresponding to the free motion) over the above momentum bins:

$$
\left|x_{i}\right\rangle=\frac{1}{\sqrt{B_{i}}} \int_{q_{i-1}}^{q_{i}} d q f(q)\left|\psi_{0 q}\right\rangle, \quad i=1, \ldots, N,
$$

where $f(q)$ is some weight function and $B_{i}$ are normalization factors, directly interrelated to each other

$$
B_{i}=\int_{q_{i-1}}^{q_{i}} d q|f(q)|^{2}
$$

The main advantage of the integration in eq. (1) is that wave-packet functions belong to $L_{2}$-space, i.e. they are normalizable in a usual sense (similarly to bound state functions) and vanishing at infinity in contrast to the initial plane waves. Thus, wave-packet functions belong to a Hilbert space. Different choices of weight function lead to different sets of WPs. In our previous calculations [14-17], we used two types of these states:

(i) energy wave-packets with weight functions $f(q)=\sqrt{\frac{q}{\mu}}$,

(ii) momentum wave-packets with constant weight function $f(q)=1$.

It should be mentioned that nevertheless different weight functions, both type of WP states are orthogonal to each other for different bins while the overlap of energy and momentum WP functions on the same bin is very close to unity (the difference from unity goes to zero when the bin width decreases). So, two type of basis functions are very close one to another. It is rather convenient to use energy WPs for spectral expansions of the operators, while the momentum ones have analytical expressions and are very useful in calculation of interaction potential matrix elements [14-17].

In the Fig. 1 coordinate behaviors of the free momentum packets (for angular momentum $L=0$ )

$$
x_{i}(r)=\sqrt{\frac{2}{\pi}} \frac{\sin (q r)}{r} \frac{\sin \left(d_{i} r / 2\right)}{d_{i} r / 2}
$$

for cases of broad, intermediate and narrow bins are given. Here $q$ is the bin midpoint and $d_{i}$ is the bin width. It is evident that these $L_{2}$-type functions are not vanishing at very far asymptotic region up to $q r \sim 200$. Thus, the wavepacket basis is very suitable for the expansion of continuous spectrum wave functions. This long-range behavior of the basis functions plays a crucial role in the three-body scattering, especially above the breakup threshold, because it provides a proper overlapping between basis functions in different Jacobi-coordinate sets.

General properties of WPs have been considered in details previously [14-17]. They form an orthonormal set. Approximations for operators in the Wave-Packet Subspace

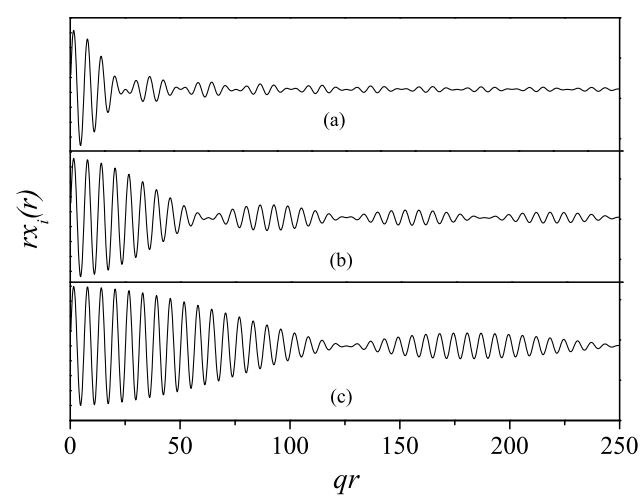

Fig. 1. The coordinate behavior of the dimensionless $s$-wave WP function $r x_{i}(r)$ for different values of the bin width $d_{i}$ (in ratio to the momentum value $q$ ): $d_{i} / q=0.25$ (a), $d_{i} / q=0.1$ (b) and $d_{i} / q=0.05(\mathrm{c})$.

(WPS) are defined by the applying the following projection operator

$$
\mathfrak{p}=\sum_{i=1}^{N}\left|x_{i}\right\rangle\left\langle x_{i}\right| .
$$

We will denote finite-dimensional (f.-d.) representations for the basic scattering operators in WPS by gothic letters.

Direct interrelation between WP's and plane wave functions allows to find explicit analytical expressions for a projection of any operator which has an explicit functional dependence of $h_{0}$. For this purpose, a spectral expansion of the operator should be used.

The most useful property of the WP basis for solution of scattering problems is the finite-dimensional representation for the resolvent of free Hamiltonian $g_{0}(E)=$ $\left[E+i 0-h_{0}\right]^{-1}$ :

$$
\mathfrak{g}_{0}(E) \equiv \mathfrak{p} g_{0}(E) \mathfrak{p}=\sum_{i=1}^{N}\left|x_{i}\right\rangle g_{i}(E)\left\langle x_{i}\right| .
$$

Here the corresponding complex-valued eigenvalues $g_{i}(E)$ have the following form:

$$
g_{i}(E)=\frac{1}{B_{i}} \int_{q_{i-1}}^{q_{i}} d q \frac{|f(q)|^{2}}{E+\mathrm{i} 0-\frac{q^{2}}{2 \mu}} .
$$

and can be evaluated analytically for the momentum and energy WP states $[15,17]$. It is clear that the imaginary part of the eigenvalues doesn't vanish only in a single interval with $i=k$ to which the respective on-shell momentum value $q$ belongs, i.e. $q \in\left[q_{k-1}, q_{k}\right]$. Although the eigenvalue (6) has logarithmic singularities if $q$ is equal to one of the singular bin endpoints, the finite-dimensional (f.d.) representation (5) for the free resolvent with eigenvalues (6) can be used directly to obtain scattering observables (we assume that energy $E$ does not coincide with any energy-bin endpoint [17] in that case). But usually the additional averaging-on-energy procedure is applied to the 


\section{$19^{\text {th }}$ International IUPAP Conference on Few-Body Problems in Physics}

eq.(6) leading to the free resolvent representation which has no singularities at all on the real energy axis [17].

In the few-body case, few-body wave-packet bases are constructed as direct productions of two-body ones. Simultaneously few-body momentum space become a discretized one. Thus, implementation of the WP basis is similar to the formulation of the few-body scattering problem in the multi-dimensional momentum lattice. That is why we call our wave-packet basis as the lattice one.

\subsection{Discrete spectral shift formalism: scattering observables from the simple diagonalization .}

Using the finite-dimensional WP analog (5) for the free resolvent one can derive easily a matrix scheme for WP solution of the Lippmann-Schwinger equation for the total Hamiltonian

$$
h=h_{0}+v,
$$

where $v$ is the short-range interaction potential from $[15$, 17]. But for the simple one-channel interaction there is another possibility of extracting scattering information directly from the diagonalization procedure for the Hamiltonian matrix in the lattice basis via the novel Discrete Spectral Shift formalism (DSS) [18].

After the diagonalization procedure for the the total Hamiltonian matrix on the free WP basis, one gets the set of eigenenergies $\left\{E_{i}\right\}_{i=1}^{N}$ and corresponding eigenfunctions $\left\{\left|z_{i}\right\rangle_{i=1}^{N}\right\} \in L_{2}$. Some part of the eigenfunctions with $i \leq N_{b}$ approximates the bound states of the system (if they exist) while the rest $\left(N-N_{b}\right)$ of the eigenfunctions can be considered as discretized (normalized) analogs of the scattering wave functions of the Hamiltonian — the so-called pseudostates.

It has been shown in our recent paper [18], that local differences of the total discretize spectrum of $h_{0}$ caused by the switching on the perturbation $v$, i.e. $h_{0} \rightarrow h=h_{0}+v$, are related to the spectral shift function $\xi(E)$. This function has been introduced in the rigorous mathematical quantum scattering theory [21-23] and is directly related to the scattering operator $S$

$$
\operatorname{det} S(E)=\exp (-2 \pi i \xi(E)) .
$$

In a single-channel case, eq.(8) implies that the SSF, within a factor $(-\pi)$, is equal to a partial phase shift:

$$
\delta(E)=-\pi \xi(E) .
$$

It has been shown $[18,20]$ that the discrete analog of the SSF, defined for the discretized spectrum of the free Hamiltonian $h_{0}$, can be found from the simpliest formula:

$$
\xi_{i}=\frac{E_{i}-E_{i}^{0}}{D_{i}}, \quad i=N_{b}+1, \ldots, N .
$$

Such dimensionless discrete shifts have been demonstrated in Ref.[18] to relate to exact continuous SSF $\xi(E)$ accurate with inverse basis dimension $N: \xi_{i}=\xi\left(E_{i}^{0}\right)+o(1 / N)$. Thus, by making use the explicit interrelation (9) between SSF and phase shifts one gets discrete approximation for phase shift as a function of energy:

$$
\delta\left(E_{i}^{0}\right)=-\pi \frac{E_{i}-E_{i}^{0}}{D_{i}}+o(1 / N), \quad i=N_{b}+1, \ldots, N
$$

Because the whole discretized spectrum of $h$ in a broad energy range, i.e. $\left\{E_{i}\right\}$, can be found from a single diagonalization of the Hamiltonian matrix, so that the eq.(11) allows to find, besides of eigenenergies and eigenfunctions of bound states, also partial phase shifts in a very broad energy range. When the basis dimension $N$ increases and the respective discretized spectrum becomes more dense we should observe convergence of the discretized phase shifts (11) to the exact ones.

So, for finding the accurate $S$-matrix in a single- and also multi-channel scattering (see below) in a broad energy range, it is not necessary at all to solve the respective single- or multi-channel scattering equations.

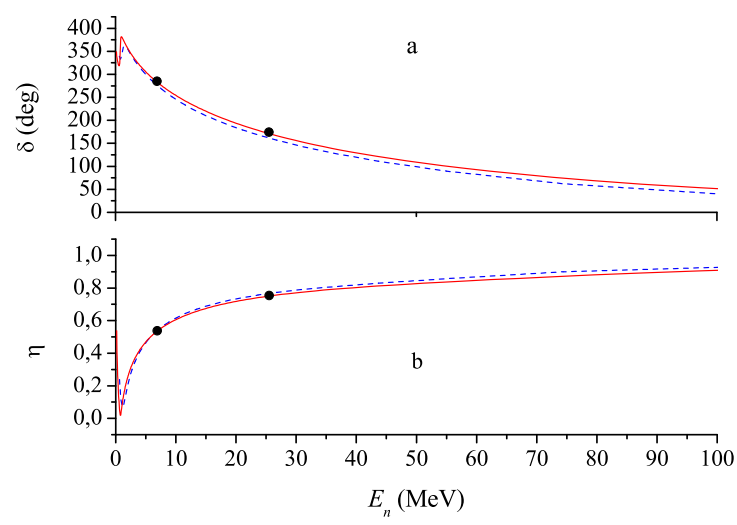

Fig. 2. Real parts of $S$-wave phase shifts $\delta$ (a) and inelasticity parameters $\eta$ (b) for neutron-nucleus scattering obtained via DSS formalism in a lattice basis with dimension $N=100$ (dashed line) and $N=200$ (full line). Black circles denote the accurate phase shifts found with local phase-shift equivalent potentials at neutron energies $E_{n}=7$ and $26 \mathrm{MeV}$.

It is important to emphasize here that the DSS method is fully applicable to a broad class of interactions. We found that it is valid even for complex-valued interaction potentials. In this case one can choose as previously, a real wavepacket basis, however the total non-Hermitian Hamiltonian eigenvalues will be complex-valued. So, the phase shifts derived from these complex eigenvalues will be also complex. As a good illustration of the applicability of the above new technique we find the complex phase shifts for the neutron scattering off a nuclear target when the $n-A$ interaction has the form of a complex non-local potential of the Perey and Buck type [19].

The complex $S$-wave phase shift $\delta_{0}(E)$ calculated from the complex-valued eigenenergies $\left\{E_{i}\right\}$ as a function of energy together with those found from the direct numerical integration of the Schrödinger equation at two energies (for 
the local phase-equivalent potentials) are displayed at Fig. 2. The potential parameters are taken the same as in the Ref. [17]. The respective angular distributions of the differential cross sections at various energies, all being found from discretized partial phase shifts for all required partial waves, are shown at Fig.3 at incident neutron energies $E_{n}=2,7,26,50 \mathrm{MeV}$. Simultaneously, we have obtained also the differential cross sections for other energies in the region $0<E \leq 200 \mathrm{MeV}$ using just a single diagonalization of the total Hamiltonian matrix at each partial wave.
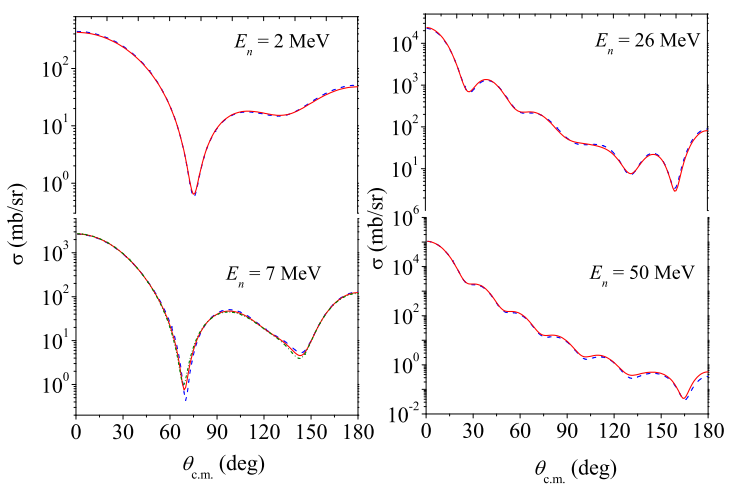

Fig. 3. Differential cross sections for neutron-nucleus scattering by a non-local complex optical potential at different energies obtained via DSS formalism in lattice bases of dimension $N=100$ (dashed line) and $N=200$ (full line). Dashed-dotted curve reflects the results for local phase-shift equivalent potential at $E_{n}=7 \mathrm{MeV}$.

\subsection{Scattering wave packets and a f.-d. representation for the resolvent of the total Hamiltonian.}

It is also possible to construct f.-d. representation for the scattering operators using pseudostate WP formalism.

Indeed, let's define complete set of eigenfunctions of the total Hamiltonian $h$ : bound states $\left\{\left|z_{n_{b}}\right\rangle\right\}_{n_{b}=1}^{N_{b}}$ and continuous set of scattering wave functions $\left|\psi_{q}\right\rangle$ normalized on $\delta$-function on $q$. Simultaneously we define some partition of the $h$ continuum (similarly to free Hamiltonian case) $\left[\bar{q}_{i-1}, \bar{q}_{i}\right]_{i=N_{b}+1}^{N}$. The scattering wave-packets can be constructed from the exact scattering wave-functions similarly as in (1):

$$
\left|z_{i}\right\rangle=\frac{1}{\sqrt{A_{i}}} \int_{\bar{q}_{i-1}}^{\bar{q}_{i}} d q w(q)\left|\psi_{q}\right\rangle, \quad i=N-N_{b}+1, \ldots, N,
$$

where $w(q)$ and $A_{i}$ are weight function and normalization factors. To distinguish these scattering wave packets from the free ones, we denote them by another letter.

The properties of the scattering WPs are absolutely the same as those of the free WPs. The full set of bound states of $h$ and scattering WPs states $\left\{\left|z_{i}\right\rangle\right\}_{i=1}^{N}$ form a basis in a
Hilbert space. Similarly to the free lattice basis, any operator commuting with Hamiltonian $h$ has a diagonal f.-d. representation in such a basis [17]. So, one has the analytical representation for the total resolvent in the scattering WP basis:

$$
\mathfrak{g}(E)=\sum_{n_{b}}^{N_{b}} \frac{\left|z_{n_{b}}\right\rangle\left\langle z_{n_{b}}\right|}{E-E_{n_{b}}}+\sum_{i=N_{b}+1}^{N}\left|z_{i}\right\rangle g_{i}(E)\left\langle z_{i}\right|,
$$

where complex eigenvalues $g_{i}(E)$ can be evaluated by the similar formula to free WPs (6)

$$
g_{i}(E)=\frac{1}{A_{i}} \int_{\bar{q}_{i-1}}^{\bar{q}_{i}} d q \frac{|f(q)|^{2}}{E+\mathrm{i} 0-\frac{q^{2}}{2 \mu}} .
$$

Further, the constructed resolvent operator can be used to find observables ( $t$-matrix) or to construct three-body channel resolvents.

The main problem in this scheme is how to construct scattering WPs $\left|z_{i}\right\rangle$ without solution of the scattering problem for the Hamiltonian $h$. The answer is that pseudostates of $h$ constructed in the previous subsection can be considered as approximations for the scattering WPs without any additional normalization factors. We found the direct correspondence between these two type of states if psedostate energy $E_{i}$ and the total Hamiltonian wave-packet eigenenergy $\left\langle z_{i}|h| z_{i}\right\rangle$ are equal one to another [15]. It is also possible to construct the partition bound-points $\bar{q}_{i}$ from the set of pseudo energies $E_{i}$ [15].

In practice, we use exact scattering WPs to find explicit analytical formula for the total resolvent while in numerical calculations we construct these scattering WPs by simple diagonalization procedure in the free lattice basis.

It should be stressed that correspondence between pseudostes and scattering WPs assists also in a case of the longrange Coulomb interaction. In the Fig.4, we compare Coulomb scattering wave packets, corresponding to the $p p$ interaction, constructed by the diagonalization of Coulomb Hamiltonian on the free WP basis with those obtained from exact Coulomb regular scattering wave functions by the formula (12). It is clear from the Figure that pseudostates are indistinguishable from the exact Coulomb WPs. This result reflects the interesting fact, that nevertheless exact regular Coulomb wave functions could not be expanded over plane waves, Coulomb WPs can be expanded over finite-dimen-sional set of the free WPs.

\section{Multi-channel scattering problem.}

It is more convenient to use in this section the energy wavepacket states. These states are constructed from the scattering (or free) wave functions $\left|\psi_{E}\right\rangle=\sqrt{\frac{\mu}{q}}\left|\psi_{q}\right\rangle$ normalized with the $\delta$-function on energy.

\subsection{Multi-channel DSS formalism.}

Usually, realistic two-body interactions are taken in multichannel form (e.g.including tensor force). Consider now 


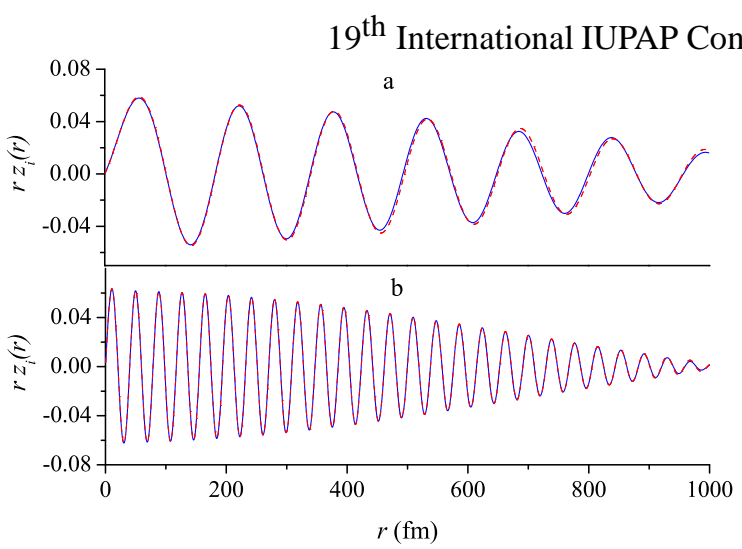

Fig. 4. Comparison of exact Coulomb wave-packets (solid curves) and Coulomb pseudostates obtained in the lattice basis (dashed curves) at two different energies $E=0.07 \mathrm{MeV}$ (a) and $E=11.3 \mathrm{MeV}$ (b) of the Coulomb $p p$ Hamiltonian.

the multi-channel scattering case with $K$ channels. The total Hamiltonian of the system can be written in a matrix form (on the channel indices)

$$
h_{v v^{\prime}}=h_{0 v} \delta_{v v^{\prime}}+v_{v v^{\prime}}, \quad v, v^{\prime}=1, \ldots, K,
$$

where $h_{0 v}$ - free channel Hamiltonians having a simple continuous spectrum in the interval $\left[\mathcal{E}_{v}, \infty\right)$ with $\mathcal{E}_{v}$ being the threshold of $v$-th channel, and $v_{v v^{\prime}}$ are the coupling potentials. The continuous spectrum of the multichannel free Hamiltonian $\mathbf{h}_{\mathbf{0}}$ is degenerate, in contrast to a single-channel case, and the degree of degeneracy $k$ is equal to the number of open channels and depends upon energy. The continuous spectrum of the total Hamiltonian $\mathbf{h}$ in the multi-channel case coincides with those of the free Hamiltonian, so that at each energy there should exist $k$ independent solutions corresponding to different boundary conditions for the initial scattering problem. Thus, the discretization procedure becomes unclear here, because when the total Hamiltonian is represented by a respective matrix in some arbitrary finite $L_{2}$ basis the required degeneration disappears, in general, and one has only one pseudostate solution at each discrete eigenenergy which can hardly be treated.

To avoid this problem in our approach, we have to arrange the partitions of spectra of the channel free Hamiltonians $h_{0 v}$ using the special condition: the discretized multichannel free Hamiltonian matrix should have degenerate eigenvalues and the corresponding degrees of degeneracy should be the same as those of the continuous spectrum of the free Hamiltonian $\mathbf{h}_{\mathbf{0}}$ [18].

To construct such a type discretization, let's divide the total continuum to $K$ regions between thresholds $\Omega_{k}=$ [ $\left.\mathcal{E}_{k}, \mathcal{E}_{k+1}\right]$ with $\mathcal{E}_{K+1} \equiv \infty$. Each part $\Omega_{k}$ of the spectrum has constant degeneracy value $k$ (we assume here that all the thresholds are different and the channels are arranged in order of increasing of the threshold energy values). Because the number of open channels in each region is preserved, we can define at each energy $E \in \Omega_{k}$ two sets of eigenfunctions of $h^{2}$ :

\footnotetext{
${ }^{2}$ Here the denote each state by its total energy $E$ which is sum
} of the kinetic energy and the threshold energy value.
- wave functions $\left\{\left|\psi_{E}^{(v)}\right\rangle\right\}_{v=1}^{k}$ corresponding to the incoming waves in the channel $v$ and

- wave-functions $\left\{\left|\tilde{\psi}_{E}^{(\varkappa)}\right\rangle\right\}_{\varkappa=1}^{k}$ defined in the so called eigenchannel representation.

The eigenchannel representation (ER) corresponds to the diagonal form of the multi-channel $S$-matrix [24]. The ER states differs from the ordinary channel states by the rotation and the rotation matrix $\mathbf{U}(E)$ depends on the energy E:

$$
\left|\psi_{E}^{(v)}\right\rangle=\sum_{\varkappa=1}^{k} U_{\nu \varkappa}(E)\left|\tilde{\psi}_{E}^{(\varkappa)}\right\rangle
$$

Matrix $\mathbf{U}(E)$ is unitary:

$$
\sum_{\varkappa=1}^{k} U_{v \varkappa}(E) U_{\varkappa v^{\prime}}^{\dagger}(E)=\delta_{v, v^{\prime}}
$$

and its dimension is equal to the number of open channels $k$.

Now one can construct the partition of the total continuum as unification of partitions of regions between thresholds. In particular, each region $\Omega_{k}$ is divided into finite number of energy bins $\left[\epsilon_{i-1, k}, \epsilon_{i, k}\right]_{i=1}^{N_{k}}$ with widths $D_{i, k} \equiv \epsilon_{i, k}-\epsilon_{i-1, k}$, where $N_{k}$ is local region partition dimensions, $\epsilon_{0, k} \equiv \mathcal{E}_{k}$ and $\epsilon_{N_{k}, k} \equiv \mathcal{E}_{k+1}$ (for the last region we confined the continuum with some sufficiently large value $E_{\max }$ and assign $\left.\mathcal{E}_{K+1}=E_{\max }\right)$.

To define energy wave-packet bases in each initial channel $v$, one should use the following partitions of the kinetic energy operators: $\bigcup_{k=v}^{K}\left[\epsilon_{i-1, k}-\mathcal{E}_{v}, \epsilon_{i, k}-\mathcal{E}_{v}\right]_{i=1}^{N_{k}}$. Using such partitions, the corresponding set of free WPs:

$\left|x_{i, k}^{(v)}\right\rangle=\frac{1}{\sqrt{D_{i, k}}} \int_{\epsilon_{i-1, k}-\mathcal{E}_{v}}^{\epsilon_{i, k}-\mathcal{E}_{v}} d \epsilon\left|\psi_{0 \epsilon}^{(v)}\right\rangle, i=1, \ldots, N_{k}, k=v, \ldots, K$

can be constructed from the plane waves $\left|\psi_{0 \epsilon}\right\rangle$, where $\epsilon$ is kinetic energy value.

It is evident, that the obtained by the above procedure discretized spectrum of the total free Hamiltonian is $k$ times degenerate in each region $\Omega_{k}$ similarly to the exact continuous spectrum of $\mathbf{h}_{0}$. The dimension of the WP basis in each channel is defined by the sum $\mathcal{N}_{v}=\sum_{k=v}^{K} N_{k}$. The total WP dimension is the following:

$$
\mathcal{N}=\sum_{v=1}^{K} \mathcal{N}_{v}=\sum_{k=1}^{K} k \cdot N_{k}
$$

It can be proven $[18,20]$, that, for the constructed spectrum, the switching on the perturbation removes the degeneracy: each $k$-fold degenerated level $E_{i, k}^{0}$ of the free Hamiltonian matrix produces a set of (non-degenerate) $k$ sublevels in the perturbed spectrum $\left\{E_{i, k}^{(\varkappa)}\right\}_{\varkappa=1}^{k}$, arranged in an increasing order. Then, each such $\varkappa$-th branch $\left\{E_{i, k}^{(\varkappa)}\right\}_{i=1}^{N_{k}}$ of the discretized spectrum can be confronted with the respective branch in the continuous spectrum of the total 
Hamiltonian in ER. So, we have a one-to-one correspondence between the discretized (quasi-continuous) spectrum of the multi-channel Hamiltonian matrix and the continuous spectrum of the initial total Hamiltonian. Due to such a classification of branches in the discretized spectrum, one can determine the parameters of the multi-channel $S$ matrix using differences between perturbed and unperturbed eigenenergies.

In this way, one can generalize the relation (10) for the case of a degenerate multi-channel spectrum and define a set of discretized spectral shift functions $\xi^{(\varkappa)}(E)$ for each spectral branch

$$
\xi_{i, k}^{(\varkappa)}=\frac{E_{i, k}^{(\varkappa)}-E_{i, k}^{0}}{D_{i, k}}, \quad \varkappa=1, \ldots, k .
$$

Further, the basic interrelation (9) between SSF and phase shift can be generalized here as

$$
\delta^{(\varkappa)}(E)=-\pi \xi^{(\varkappa)}(E), \quad \varkappa=1, \ldots, k,
$$

where the $\delta^{(\varkappa)}(E)$ are the so-called eigenphases, or the phase shifts in the (ER) and the energy $E$ belongs to the region where the continuous spectrum is $k$-fold degenerate [18]. As a result, one gets a simple formula for the phase shifts of the multi-channel $S$-matrix in ER :

$$
\delta^{(\varkappa)}\left(E_{i, k}^{0}\right) \approx-\pi \frac{E_{i, k}^{(\varkappa)}-E_{i, k}^{0}}{D_{i, k}}, \varkappa=1, \ldots, k .
$$

It is important to stress here that the main interrelation (21) between multi-channel SSFs $\xi^{(\varkappa)}(E)$ and eigenphases does not follow from the original Birman-Krein formula (8) but should be considered just as its generalization. The crucial point here is that we introduce different "channel" SSFs $\xi^{(\varkappa)}(E)$ for each $\varkappa$-th branch of the multi-channel spectra in contrast with a conventional definition of single SSF $\xi(E)$. The present authors are unaware about any previous works devoted to such generalization of SSF formalism to the multi-channel case but we made sure in validity of the relation (22) for the phase shifts by numerous examples.

Thus, the knowledge of phase shifts in ER is quite sufficient for finding total scattering cross section because the $S$-matrix in its own ER is diagonal with the following eigenvalues:

$$
S_{\varkappa}=\exp \left(2 i \delta^{(\varkappa)}\right)
$$

However for many applications one needs just $S$-matrix elements in the representation of the initial channels $S_{v v^{\prime}}$. At every energy, these matrix elements are related to the diagonal matrix elements $S_{\varkappa}$ in ER by the same rotation matrix $\mathbf{U}(E)$ as in (16):

$$
S_{\nu v^{\prime}}(E)=\sum_{\varkappa} S_{\varkappa}(E) U_{\varkappa v}(E) U_{\varkappa \nu^{\prime}}^{*}(E) .
$$

In our discretization scheme, we have discretized on energy analogs for the rotation matrix, i.e. set of matrices $\mathbf{U}^{i, k}$. To determine elements of these matrices one needs to use the expansion coefficients for the discrete pseudostates (at given energy, i.e. having sub-index $i, k$ ) over the

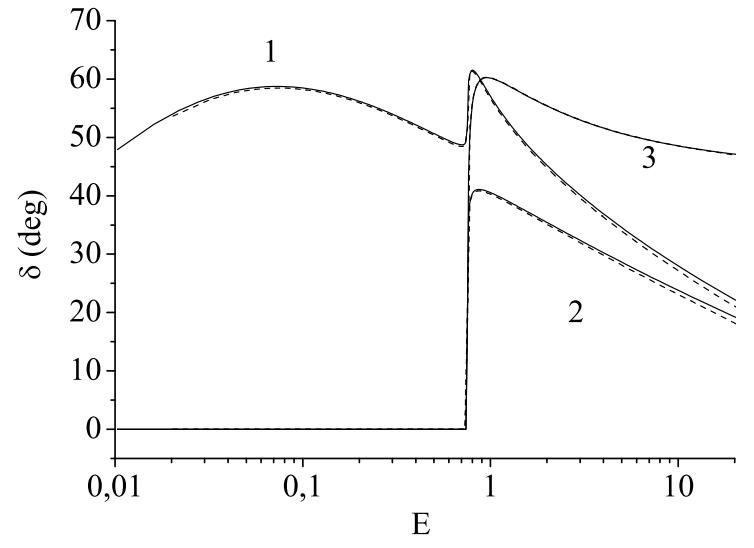

Fig. 5. Eigen phase shifts for the 1st (1) and 2nd (2) channels and the mixing angle (3) for the potential (27) obtained in the lattice basis of dimensions $\left(N_{1}+N_{2}\right):(100+50)$ - dashed curve and $(200+100)$ - full curve.

channel basis functions (18)

$$
\left|z_{i, k}^{(\varkappa)}\right\rangle=\sum_{k^{\prime}=1}^{K} \sum_{v=k^{\prime}}^{K} \sum_{j=1}^{N_{k^{\prime}}} C_{i k, j k^{\prime}}^{(\varkappa, v)}\left|x_{j, k^{\prime}}^{(v)}\right\rangle .
$$

By making use of straightforward matrix algebra one can show that the rotation matrix elements can be found from the formula

$$
U_{\varkappa \nu}^{i, k} U_{\varkappa \nu^{\prime}}^{* i, k}=\sum_{k^{\prime}=\varkappa}^{K} \sum_{j=1}^{N_{k^{\prime}}} C_{j k^{\prime}, i k}^{(\varkappa, v)} C_{j k^{\prime}, i k}^{*\left(\chi, v^{\prime}\right)} .
$$

So that, by using the presented formalism for the discrete phase shifts in the lattice basis one can derive all the elements of the complete multi-channel $S$-matrix in a wide energy range using just a single diagonalization of the total Hamiltonian matrix.

To illustrate the workability and efficiency of the above DSS method we use the Burke potential which is model for the two-channel electron-hydrogen scattering (see for example [25]):

$$
V_{11}=V_{22}=-1.5 \frac{\mathrm{e}^{-r}}{r}, \quad V_{12}=-0.25 \mathrm{e}^{-r} .
$$

The second channel threshold is shifted to the value $\Delta_{2}=$ $0.75\left(\Delta_{1}=0\right)$. At energy $E>\Delta_{2}$ the continuum is two times degenerated. In the Fig. 5 the eigenphases and the mixing parameter for the potential (27) obtained by formulas (22) and (26) are given [18].

Also we found the $S$-matrix element and corresponding elastic and reaction cross sections for $1 \rightarrow 1$ transitions. They are displayed in the Fig.6. For the comparison we add to this figure the results of the Ref. [25] (black circles) obtained by the reduction of initial two-channel problem to the one-channel one with complex non-local potential. 


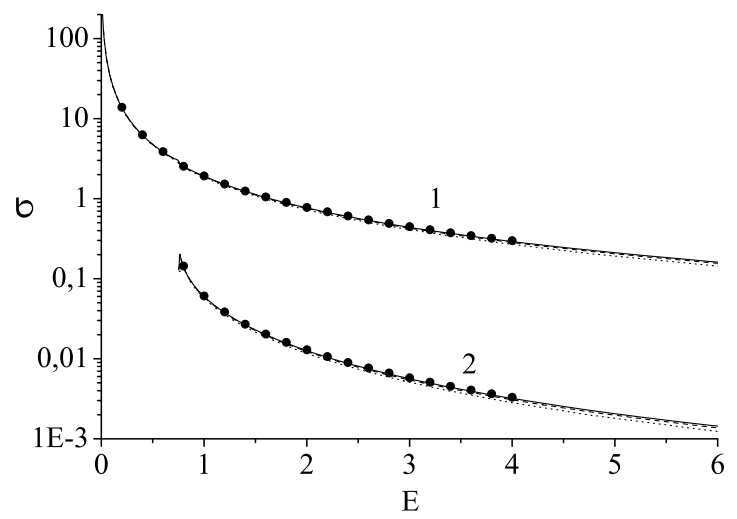

Fig. 6. Elastic cross section $\sigma_{\text {el }}(1)$ and the reaction cross section $\sigma_{\mathrm{r}}(2)$ for the potential (27)obtaiden by the DSS formalism with bases of dimension: $(50+25)$ - dotted curve, $(100+50)-$ dashed curve, $(200+100)$ - full curve. Black circles $(\bullet)$ reflects the results of the Ref. [25].

As is evident from Fig.6, our fully discretized approach based on the SSF gives quite accurate results for realistic two-channel $S$-matrix.

\subsection{Representation for the multi-channel resolvent.}

It is also possible to derive the analytical f.-d. representation for the total multi-channel resolvent (similar to onechannel case) using just the new treatment of the multichannel pseudostates in the lattice basis.

Indeed, similarly to the one-channel case we can relate pseudostates in the ER with the multi-channel scattering wave packets. For this purpose, we define now multichannel scattering wave-packets as integrals of the scattering wave-functions in ER similarly to the one channel case:

$$
\left|z_{j, k}^{(\varkappa)}\right\rangle=\frac{1}{\sqrt{A_{j, k}}} \int_{E_{j-1, k}}^{E_{j, k}} d E\left|\tilde{\psi}_{E}^{(\varkappa)}\right\rangle
$$

Now we should derive the spectral expansion of the total multi-channel resolvent using ER scattering states. The spectral expansion of the resolvent over the 'ordinary' set of the initial channel eigen functions of $h$ can be written as sum of bound-state and continuum parts $g(E)=$ $g_{b}(E)+g_{c}(E)$, where

$$
g_{b}(E)=\sum_{n_{b}=1}^{N_{b}} \frac{\left|\psi_{n_{b}}\right\rangle\left\langle\psi_{n_{b}}\right|}{E-E_{n_{b}}}, g_{c}(E)=\sum_{v=1}^{K} \int_{\Delta_{v}}^{\infty} d q^{\prime} \frac{\left|\psi_{q^{\prime}}^{(v)}\right\rangle\left\langle\psi_{q^{\prime}}^{(v)}\right|}{E+\mathrm{i} 0-E^{\prime}}
$$

Further, the $g_{c}(E)$ part can be written in the form of a sum of integrals over the regions $\Omega_{k}$ :

$$
g_{c}(E)=\sum_{k=1}^{K} \sum_{\nu=1}^{k} \int_{\mathcal{E}_{k}}^{\mathcal{E}_{k+1}} d E^{\prime} \frac{\left|\psi_{E^{\prime}}^{(v)}\right\rangle\left\langle\psi_{E^{\prime}}^{(v)}\right|}{E+\mathrm{i} 0-E^{\prime}}
$$

where $E^{\prime}$ denotes total energy of the state. It is easy to show, using the property (17), that the following relation is

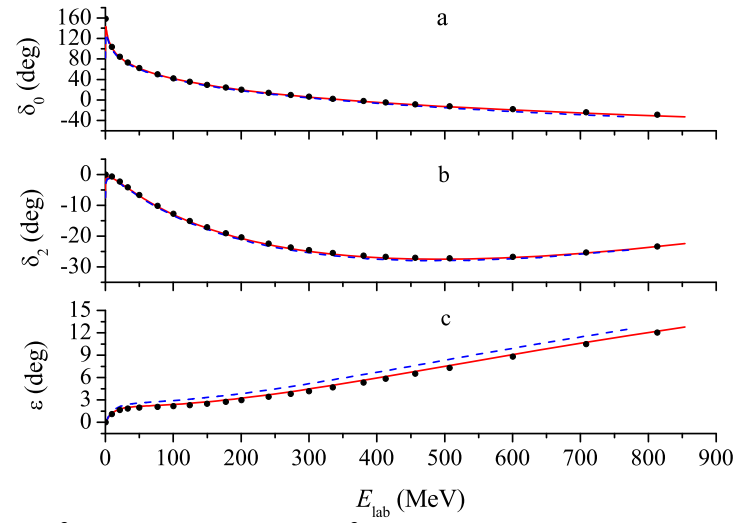

Fig. 7. ${ }^{3} S_{1}$ phase shifts $\delta_{0}$ (a), ${ }^{3} D_{1}$ phase shifts $\delta_{2}$ (b) and mixing angle $\epsilon$ (c) for the coupled-channel NN potential [26] calculated via multi-channel pseudostate formalism in WP bases of dimension $N=50$ (dashed line) and $N=100$ (full line) in each channel. Black circles represent results of direct numerical integration of the Lippmann-Schwinger equation at each required energy.

valid:

$$
\sum_{v=1}^{k}\left|\psi_{E^{\prime}}^{(v)}\right\rangle\left\langle\psi_{E^{\prime}}^{(v)}\left|=\sum_{\varkappa=1}^{k}\right| \tilde{\psi}_{E^{\prime}}^{(\varkappa)}\right\rangle\left\langle\tilde{\psi}_{E^{\prime}}^{(\varkappa)}\right| .
$$

Thus, we come to the spectral expansion of the continuum part of the total resolvent over Hamiltonian eigen functions in ER:

$$
g_{c}(E)=\sum_{k=1}^{K} \sum_{\chi=1}^{k} \int_{\mathcal{E}_{k}}^{\mathcal{E}_{k+1}} d E^{\prime} \frac{\left|\tilde{\psi}_{E^{\prime}}^{(\varkappa)}\right\rangle\left\langle\tilde{\psi}_{E^{\prime}}^{(\varkappa)}\right|}{E+\mathrm{i} 0-E^{\prime}}
$$

Applying the discretization procedure one gets the following f.-d. representation for the total multi-channel resolvent over multi-channel WPs

$$
\mathfrak{g}_{c}(E)=\sum_{k=1}^{K} \sum_{j=1}^{N_{k}} g_{j, k}(E)\left\{\sum_{\varkappa=1}^{k}\left|z_{j, k}^{(\varkappa)}\right\rangle\left\langle z_{j, k}^{(\varkappa)}\right|\right\}
$$

where complex eigenvalues are defined as integrals

$$
g_{j, k}(E)=\frac{1}{D_{j, k}} \int_{\epsilon_{j-1, k}}^{\epsilon_{j, k}} d E^{\prime} \frac{1}{E+\mathrm{i} 0-E^{\prime}} .
$$

It looks like the multi-channel pseudostates, which we obtained in the previous subsection can be considered as approximations to the multi-channel WPs. This fact allows to simplify drastically the evaluation of the channel threebody resolvents in the case of multi-channel two-body subsystems.

As illustration we calculate the coupled-channel ${ }^{3} S_{1}$ ${ }^{3} D_{1}$ partial phase shifts and the mixing angle $\epsilon$ for a realistic $N N$ potential [26] with tensor force in the triplet channel: $S=1, I=0$. The two-channel $T$ matrix has been obtained from the formula $t(E)=v+v g(E) v$, where f.d. representation for the resolvent (33) in the two-channel pseudostate basis has been used. In the Fig. 7 the phase shifts $\delta_{0}(E), \delta_{2}(E)$, and mixing parameter $\epsilon_{1}(E)$ (i.e. those which enter the $N N$ differential scattering cross sections - 
in the Stapp parametrization) are shown in very wide energy range $0<E_{\text {lab }}<900 \mathrm{MeV}$. They are found from just a single diagonalization of the respective two-channel Hamiltonian of the $N N$ interaction. We compare them to values obtained from direct numerical solutions of the twochannel Lippmann-Schwinger equation at many energies in the above energy range.

\section{Solution of the general three-body scattering problem}

Here we will briefly describe how the wave-packet technique can be applied to the general three- (or few-) body scattering problem with any type of interaction potentials including local, non-local etc. ones by the introduction of the two-dimensional momentum lattice.

\subsection{Lattice representation for the channel resolvent}

Let's consider the general three-body scattering problem for particles 1,2 and 3, interacting via pair short-range potentials $v_{i}(i=1,2,3)$. It is convenient to use three Jacobi coordinate sets corresponding to three channel Hamiltonians $H_{i}(i=1,2,3)$ which define asymptotic motions in the system. In the general case the respective wave-packet basis should be constructed independently for each Jacobi set [17].

The channel Hamiltonian $H_{1}$ has the form of the direct sum of the two-body subHamiltonians

$$
H_{1} \equiv h_{1} \oplus h_{0}^{1},
$$

where subHamiltonian $h_{1}$ defines the interaction in the $\{23\}$ subsystem (i.e. including the potential $v_{1}$ ) and the subHamiltonian $h_{0}^{1}$ corresponds to the free relative motion of this subsystem (its center of mass) and the spectator particle 1 . The eigenfunction of the three-body Hamiltonian $H_{1}$ corresponding to the given value of the total angular momentum $\boldsymbol{\Lambda}$ and its projection $\mathcal{M}$ can be written in the following form:

$$
\left\langle\mathbf{p}^{\prime}, \mathbf{q}^{\prime} \mid \psi_{p}^{l}, \psi_{0 q}^{L}, \Lambda \mathcal{M}\right\rangle \equiv \psi_{p}^{l}\left(p^{\prime}\right) \psi_{0 q}^{L}\left(q^{\prime}\right) \mathcal{Y}_{l L}^{\Lambda \mathcal{M}}\left(\hat{\mathbf{p}}^{\prime}, \hat{\mathbf{q}}^{\prime}\right),
$$

where $\mathbf{p}^{\prime}$ and $\mathbf{q}^{\prime}$ are the relative and c.m. Jacobi momenta; $\psi_{p}^{l}\left(p^{\prime}\right)$ and $\psi_{0 q}^{L}\left(q^{\prime}\right)$ are the radial wave functions corresponding to the subHamiltonians $h_{1}$ and $h_{0}^{1}$ with angular momenta $\mathbf{I}$ and $\mathbf{L}$ respectively. The $\mathcal{Y}_{l L}^{\Lambda \mathcal{M}}\left(\hat{\mathbf{p}}^{\prime}, \hat{\mathbf{q}}^{\prime}\right)$ are six-dimensional spherical harmonics given by the convolution of the spherical functions $Y_{l \mu_{1}}\left(\hat{\mathbf{p}}^{\prime}\right)$ and $Y_{L \mu_{2}}\left(\hat{\mathbf{q}}^{\prime}\right)$.

Now we introduce the WP bases for two-body subHamiltonians $h_{1}$ and $h_{0}^{1}$. Assume further there are $K_{\mathrm{b}}^{l}$ bound states (for a fixed partial wave $l$ ) in the $\{23\}$ subsystem with corresponding bound-state wave functions $\left\{\left|z_{n}^{l}\right\rangle\right\}_{n=1}^{K_{\mathrm{b}}^{l}}$ and eigen energies $\left\{\epsilon_{n}^{l *}\right\}_{n=1}^{K_{\mathrm{b}}^{l}}$. Let's define the partition $\left[\epsilon_{i-1}^{l}, \epsilon_{i}^{l}\right]_{i=K_{\mathrm{b}}^{l}+1}^{K^{l}}$ of the continuous spectrum of $h_{1}$ (with corresponding momentum bins $\left.\left[p_{i-1}^{l}, p_{i}^{l}\right]_{i=K_{\mathrm{b}}^{l}+1}^{K_{l}}\right)$ and construct the set of the scattering wave packets (12) from the respective exact scattering wave functions $\left|\psi_{p}^{l}\right\rangle$. The full lattice basis $\left\{\left|z_{i}^{l}\right\rangle\right\}_{i=1}^{K_{l}}$ for the $h_{1}$ subHamiltonian includes the bound state functions and the scattering wave packets. Free wave packets (1) corresponding to the $h_{0}^{1}$ subHamiltonian are constructed with the usage of the partition $\left[q_{j-1}^{L}, q_{j}^{L}\right]_{j=1}^{N_{L}}$ in the continuous spectrum of the $h_{0}^{1}$.

Now one can build the three-body wave-packets (3WP) just as products of two type wave-packet states (for the above two subHamiltonians) whose angular parts are combined to the total angular momentum value $\Lambda$ similarly to eq.(36):

$$
\left|Z_{S}^{\Lambda \mathcal{M}}\right\rangle \equiv\left|z_{i}^{l}, x_{j}^{L}, \Lambda \mathcal{M}\right\rangle, \quad i=1, \ldots, K_{l}, \quad j=1, \ldots, N_{L},
$$

where $S=i, l, j, L$ is the multi-index. Further, we will omit the index $\mathcal{M}$. The properties of the $3 \mathrm{WP}$ constructed in this way are the same as properties of the two-body wave packets, viz. they form an orthonormal set and any operator functionally dependent of the channel Hamiltonian $H_{1}$ has the diagonal projection onto the subspace spanned on this basis. It allows us to construct an analytical f.-d. approximation for the three-body channel resolvent $G_{1}(E) \equiv$ $\left[E+\mathrm{i} 0-H_{1}\right]^{-1}$.

Indeed, the exact three-body channel resolvent is the convolution of the two-body subresolvents $g_{1}(E)$ and $g_{0}(E)$ :

$$
G_{1}(E)=\frac{1}{2 \pi \mathrm{i}} \int_{-\infty}^{\infty} \mathrm{d} \epsilon g_{1}(\epsilon) g_{0}(E-\epsilon) .
$$

Using spectral expansions for the two-body resolvents and performing the integration, one gets an explicit expression for the exact channel resolvent $G_{1}$ as a sum of two terms $G_{1}(E)=G_{1}^{\mathrm{BC}}(E)+G_{1}^{\mathrm{CC}}$, where the bound-continuum part takes the form:

$$
G_{1}^{\mathrm{BC}}(E)=\sum_{l, L} \sum_{n=1}^{K_{\mathrm{b}}^{l}} \int_{0}^{\infty} \mathrm{d} q \frac{\left|z_{n}^{l}, \psi_{0 q}^{L}, \Lambda\right\rangle\left\langle z_{n}^{l}, \psi_{0 q}^{L}, \Lambda\right|}{E+\mathrm{i} 0-\epsilon_{n}^{l *}-\frac{q^{2}}{2 M}},
$$

where $M$ is the reduced mass in the $\{23\}+1$ channel. The continuum-continuum part of $G_{1}$ takes the form:

$$
G_{1}^{\mathrm{CC}}(E)=\sum_{l, L} \int_{0}^{\infty} \mathrm{d} p \int_{0}^{\infty} \mathrm{d} q \frac{\left|\psi_{p}^{l}, \psi_{0 q}^{L}, \Lambda\right\rangle\left\langle\psi_{p}^{l}, \psi_{0 q}^{L}, \Lambda\right|}{E+\mathrm{i} 0-\frac{p^{2}}{2 \mu}-\frac{q^{2}}{2 M}}
$$

where $\mu$ is the reduced mass in the $\{23\}$ subsystem.

Projecting further the exact channel resolvent onto $3 \mathrm{WP}$ basis defined in eq. (37), one can find the following analytical formulas for the diagonal f.-d. approximation of the $G_{1}$ operator:

$$
\begin{array}{r}
\mathfrak{5}_{1}^{\mathrm{BC}}=\sum_{l, L} \sum_{n=1}^{K_{\mathrm{b}}^{l}} \sum_{j=1}^{N_{L}} G_{n l j L}^{B C}\left|z_{n}^{l}, x_{j}^{L}, \Lambda\right\rangle\left\langle z_{n}^{l}, x_{j}^{L}, \Lambda\right|, \\
\mathfrak{5}_{1}^{\mathrm{CC}}=\sum_{i=K_{\mathrm{b}}^{l}+1}^{K_{l}} \sum_{j=1}^{N_{L}} G_{i l j L}^{C C}\left|z_{i}^{l}, x_{j}^{L}, \Lambda\right\rangle\left\langle z_{i}^{l}, x_{j}^{L}, \Lambda\right|,
\end{array}
$$




\section{$19^{\text {th }}$ International IUPAP Conference on Few-Body Problems in Physics}

where the matrix elements $G_{n l j L}^{\mathrm{BC}}$ and $G_{i l j L}^{\mathrm{CC}}$ are defined as integrals over the respective momentum bins:

$$
\begin{gathered}
G_{n l j L}^{B C}=\frac{1}{B_{j}^{L}} \int_{q_{j-1}^{L}}^{q_{j}^{L}} \frac{|f(q)|^{2} \mathrm{~d} q}{E+\mathrm{i} 0-\epsilon_{n}^{l *}-\frac{q^{2}}{2 M}}, \\
G_{i l j L}^{C C}=\frac{1}{A_{i}^{l} B_{j}^{L}} \int_{p_{i-1}^{l}}^{p_{i}^{l}} \int_{q_{j-1}^{L}}^{q_{j}^{L}} \frac{|w(p)|^{2}|f(q)|^{2} \mathrm{~d} p \mathrm{~d} q}{E+\mathrm{i} 0-\frac{p^{2}}{2 \mu}-\frac{q^{2}}{2 M}} .
\end{gathered}
$$

These matrix elements depend, in general, on the spectrum partition parameters (i.e. $p_{i}^{l}$ and $q_{j}^{L}$ values) and the total energy only and do not depend explicitly on the interaction potential $v_{1}$. When the wave-packet expansions of the three-body amplitude is convergent, the final result turns out to be independent upon the particular spectral partition parameters. The integrals in eqs.(41a) and (41b) can be taken in a closed form which gives a convenient analytical f.-d. representation for the three-body channel resolvent $G_{1}$. We have calculated these matrix elements for the case of energy packets [17].

The representation (41) for the channel resolvent is the basic feature for the wave-packet approach since it allows to simplify solution of the general three-body scattering problem drastically. In particular, this representation has been used to solve the f.-d. analog for the Faddeev equations for the Faddeev components of the total scattering wave function [16]. Alternatively this representation has been used also to solve some particular three-body scattering problems with employment of the three- (or few-) body Lippmann-Schwinger equations [14], e.g. for composite projectile scattering off nuclear target (see below) or for electron-atom and electron-molecule scattering above the first ionization threshold etc.

\subsection{Application to the general Faddeev equations.}

In case when rearrangement channels cannot be neglected the general Faddeev formalism should be used to solve the full three-body scattering problem of $2 \rightarrow 2$ or $2 \rightarrow 3$ type. So, in the Faddeev approach we introduce three sets of 3WPs $\left\{Z_{S}^{(a)}\right\}, a=1,2,3$ related to three channel Hamiltonians $H_{a}{ }^{3}$. Further, we apply the complete discretization procedure directly to the system of three Faddeev equations (e.g. for the wavefunction with the initial state $\left|\Phi_{01}\right\rangle$ defined by the channel Hamiltonian $H_{1}$ ):

$$
\left|\psi^{(a)}\right\rangle=\left|\Phi_{01}\right\rangle \delta_{a 1}+G_{a} v_{a} \sum_{b \neq a}\left|\psi^{(b)}\right\rangle, \quad a=1,2,3 .
$$

Now, in the wave-packet approach we replace each Faddeev component $\left|\psi^{(a)}\right\rangle$ with its projection onto $3 \mathrm{WP}$ basis in the channel $(a)$ :

$$
\left|\hat{\psi}^{(a)}\right\rangle=\sum_{S} O_{S}^{a}\left|Z_{S}^{(a)}\right\rangle, \quad a=1,2,3,
$$

\footnotetext{
${ }^{3}$ We omit in this subsection partial wave indices
}

and then one replaces exact channel resolvents $G_{a}=(E-$ $\left.H_{a}\right)^{-1}$ with their f.-d. 3WP analogues given in eq. (41). Finally, one gets the following f.-d. equations for the Faddeev components of the "packetized" wavefunction:

$$
\left|\hat{\psi}^{(a)}\right\rangle=\left|Z_{S_{0}}^{(1)}\right\rangle \delta_{a 1}+\left(\mathfrak{5}_{a} \mathfrak{v}_{a} \sum_{b \neq a}\left|\hat{\psi}^{(b)}\right\rangle, \quad a=1,2,3,\right.
$$

where $\left|Z_{S_{0}}^{(1)}\right\rangle$ is the $3 \mathrm{WP}$ state corresponding to the initial state $\left|\Phi_{01}\right\rangle$. One of the main advantages of the momentumlattice scheme here is that the transformation between components in the different Jacobi sets can be expressed by a f.-d. matrix of the "permutation operator", i.e.

$$
\left\langle\hat{\psi}^{(b)} \mid \hat{\psi}^{(a)}\right\rangle=\sum_{S, S^{\prime}} O_{S}^{a *} O_{S^{\prime}}^{b} P_{S, S^{\prime}}^{a b}, \quad P_{S, S^{\prime}}^{a b} \equiv\left\langle Z_{S}^{(a)} \mid Z_{S^{\prime}}^{(b)}\right\rangle,
$$

in contrast to the direct solving the Faddeev equations in the momentum space, where time-consuming multi-dimensional interpolations for scattering solution should be taken in different Jacobi coordinates are required in each step of the iteration. Using the expansion of the scattering packets $\left|Z_{S}^{(a)}\right\rangle$ on the wave-packet basis, one can express the above permutation matrix $\mathbf{P}^{a b}$ in terms of the overlap matrix for free three-body packets in different Jacobi coordinates $P_{k j, k^{\prime} j^{\prime}}^{0 a b} \equiv\left\langle x_{k}, x_{j},(a) \mid x_{k^{\prime}}, x_{j^{\prime}},(b)\right\rangle$ which do not depend on the interaction and can be calculated easily (each matrix element can be found through a one-dimensional numerical integration [16]).

Thus, the wave-packet approach can provide direct solutions for the general Faddeev equations for any two-body interactions at the real energy and without any deformation of integration path. So, the WP technique with momentum lattice representation looks quite universal and convenient for practical applications to general three-body scattering problems.

\subsection{Solution of the $n-d$ scattering problem}

As illustration of the effectiveness for the above lattice technique, we calculated the real phase shifts and inelasticity parameters for the three-body elastic $n-d$ scattering in the quartet and doublet $S$-wave channels with model Malfliet-Tjon $N N$ potential [16]. The results of these calculations are shown in Figs. 8 and 9 for the spin-quartet channel.

To check the accuracy of the method we have compared our results with the the previous benchmark calculation results from ref. [27] (below the deuteron breakup threshold) and ref. [28] (above the deuteron breakup threshold) The parameters of the $N N$ potential are taken from ref. [28].

As it is seen from the Figs. 8-9 the wave-packet discretization technique for the three-body continuum works successfully for the general three-body scattering problem both below and above breakup threshold. Thus, at the first time we have solved the three-body scattering problem above the break-up threshold using f.-d. approximation of the $L_{2}$ type for the Faddeev kernel. Just the use of the momentumlattice basis allowed us to achieve a good convergence and accuracy on this way. 


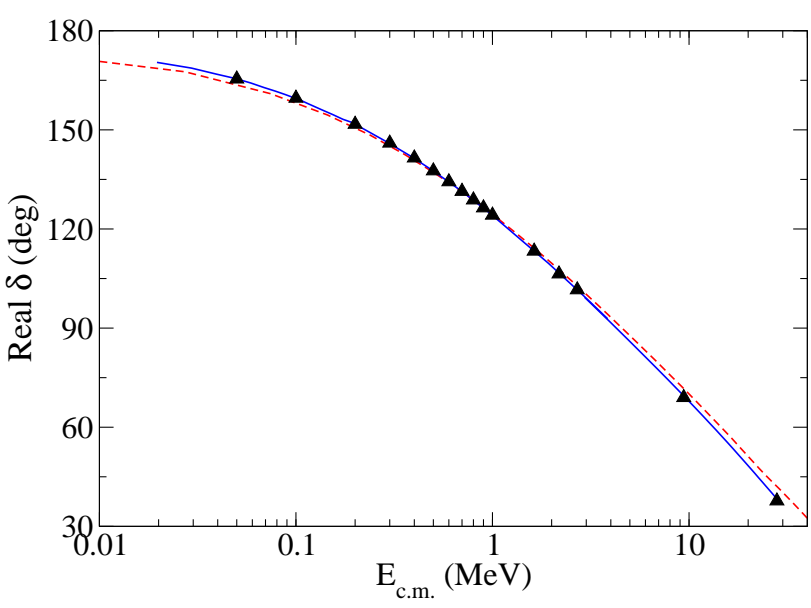

Fig. 8. The energy dependence of the real phase shift for $S$-wave quartet $n d$ scattering calculated by means of momentum-packet discretized Faddeev equation at different dimensions $M \times N$ of the lattice basis: $100 \times 100$ (dashed curve), $200 \times 200$ (solid curve). Results of the direct Faddeev equation solution from ref.[27,28] are marked as $\mathbf{\Lambda}$.

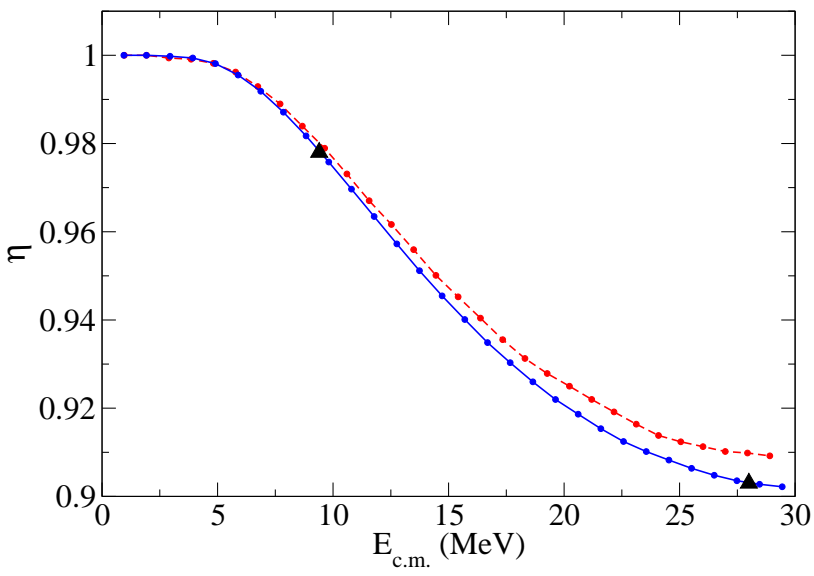

Fig. 9. The same as in Fig. 8 but for the inelasticity parameter.

\section{Conclusion}

We have demonstrated here that the formulation of the quantum scattering problems in terms of a wave-packet lattice basis is a very convenient language of discretization and an effective tool for practical solutions. Along with the main results explained in this paper, the wave-packet approach has a wide field of application: very economical solving coupled-channel problems for atomic, molecular and nuclear physics cases [14], construction of effective optical potentials of composite particle interaction [29] etc.

It should be mentioned, that although the above lattice wave-packet technique seems to be close to well known lattice approach in the effective field theory [30] it is essentially different. The main difference relates to the implementation of the special wave-packet basis to make the discretization of the momentum space in our approach. So all the operators are represented by matrices, we do not need in a formulation of asymptotic boundary conditions on the lattice. As a result, the WP formalism do not depend on the lattice geometry and mesh-point distributions. Recently developed Discrete Spectral Shift formalism in the lattice basis allows to find phase shifts from the differences of perturbed and unperturbed eigen energies of the lattice states, which looks very similar to Lüscher finite-volume formula [30] for phase shifts in the lattice EFT. But the DSS scheme seems to be more general, because it follows from the spectral shift formalism and remains the same for different lattice geometry. Moreover, it fully applicable for the multi-channel scattering.

\section{Acknowledgements}

The authors appreciate greatly a partial financial support from the DFG grant 436RUS1 13/790/0-2, the RFBR grants 08-02-91959 and 07-02-00609 and the Russian President grant MK-202.2008.2

\section{References}

1. A. Deltuva, A.C. Fonseca, Phys. Rev. C 76, 021001(R) (2007).

2. R. Lazauskas, J. Carbonell, Phys. Rev. C 70, 044002 (2004).

3. H. Witala et. al, Phys. Rev. C 63, (2001) 024007.

4. V.M. Suslov and B. Vlahovic, Phys. Rev. C 69, (2004) 044003.

5. E.J. Heller, Phys. Rev. A 12, (1975) 1222.

6. J.R. Winick, W.P. Reinhardt, Phys. Rev. A 18, (1978) 910,ibid. (1978) 925.

7. H.A. Yamani, M.S. Abdelmonem, J. Phys. B 30, (1997) 1633.

8. A.S. Kadyrov, I.Bray, Phys. Rev. A 66, (2002) 012710.

9. I. Cacelli et al., Phys. Rev. A 57, (1998) 1895.

10. Z. Papp et al., Phys. Rev. A 63, (2001) 062721; P. Doleschall, Z. Papp, Phys. Rev. C 72, (2005) 044003.

11. J.M. Bang et al., Ann. Phys. 208, (2000) 299.

12. S. Quaglioni et al., Phys. Rev. C 69, (2004) 044002.

13. R. Kozack, F.S. Levin, Phys. Rev. C 36, (1987) 883.

14. O.A. Rubtsova et al., Phys. Rev. C 78, (2008) 034603.

15. V.I. Kukulin, O.A. Rubtsova, Theor. Math. Phys. 130 , 54 (2002); ibid. 150, 403 (2007).

16. V.N. Pomerantsev et al., Phys. Rev. C 79, (2009) 034001.

17. O.A. Rubtsova et al., Phys. Rev. C 79, (2009) 064602.

18. V.I. Kukulin et al., JETP letters 90, (2009) 402.

19. F.Perey and B. Buck, Nucl. Phys. 32, (1962) 353.

20. I.M. Lifshitz, JETP, 17, (1947) 1017; ibid. 17, (1947) 1076.

21. I.M. Lifshitz, Uspekhi Math. Nauk 17, (1952) 171.

22. M.S.Birman, M.G.Krein, Sov. Math. Dokl. 3, (1962) 740.

23. V.S.Buslaev, L.D.Faddeev, Sov. Math. Dokl. 1, 451 (1960).

24. M. Danos, W. Greiner, Phys. Rev. 146, (1966) 708.

25. B. H. Bransdent and A. T. Stelbovics, J. Phys. B: At. Mol. Phys. 17, (1984)1877. 


\section{$19^{\text {th }}$ International IUPAP Conference on Few-Body Problems in Physics}

26. V.I.Kukulin, V.N.Pomerantsev, A.Faessler, Phys. Rev. C 59, 3021 (1999).

27. C.R.Chen et al., Phys. Rev. C 39, (1989) 1261.

28. J.L. Friar et al., Phys. Rev. C 42, (1990) 1838.

29. O.A. Rubtsova et al., Bulletin of Russian Academy of Sciences: Physics, 73, (2009) 750.

30. M. Lüscher, Nucl. Phys. B354, (1991) 531. 